\title{
Length Spectrum Theory, Non-backtracking Cycles, and Two Graph Analysis Tasks
}

\author{
Tina Eliassi-Rad \\ tina@eliassi.org \\ Northeastern University \\ Boston, MA
}

\section{ABSTRACT}

Two basic tasks in graph analysis are: (1) computing the distance between two graphs and (2) embedding of the graph elements (i.e., nodes or links) into a lower-dimensional space. The former task has numerous applications from $k$-nearest neighbor search, to clustering a collection of graphs, to transfer learning. Unfortunately, there exists no canonical way of computing the distance between twofi nite graphs because the dissimilarity problem is ill-defined. Despite this fact, the relevant literature contains many methods for measuring graph distance with different heuristics, computational efficiency, interpretability, and theoretical soundness [1]. We introduce a graph distance, called the Non-Backtracking Spectral Distance (NBD), which is theoretically sound and interpretable. NBD is based on a mathematical construct from algebraic topology (in particular, homotopy) called the length spectrum. The length spectrum of a graph is a function defined on the graph'sfirst homotopy group (a.k.a. the fundamental group); and it uniquely characterizes a graph's 2-core up to isometry [2]. ${ }^{1}$ Thus, if two graphs have the same length spectra, then their 2-cores are isometric. We show the relationship between a graph's length spectrum and its non-backtracking cycles; and present a method based on computing the eigenvalues of a graph's non-backtracking matrix. For the second task (i.e., graph embedding), most existing methods are stochastic and depend on black-box models such as deep networks. Both of these characteristics make their output difficult to analyze. We propose the Non-Backtracking Embedding Dimensions (NBED) forfi nding a graph embedding in low-dimensional space by computing the eigenvectors of the non-backtracking matrix. Both NBD and NBED are interpretable in terms of features of complex networks such as hubs, triangles, edge centrality, and communities. We showcase the usefulness of both NBD and NBED in experiments on synthetic and real-world networks.
\end{abstract}

This is joint work with Leo Torres at Northeastern University and Pablo Suárez Serrato at Universidad Nacional Autónoma de México.

\footnotetext{
${ }^{1}$ The $k$-core of a graph is the maximal subgraph in which each node in the subgraph
} has degree at least $k$.

Permission to make digital or hard copies of part or all of this work for personal or classroom use is granted without fee provided that copies are not made or distributed for profit or commercial advantage and that copies bear this notice and the full citation on thefi rst page. Copyrights for third-party components of this work must be honored

For all other uses, contact the owner/author(s).

GRADES-NDA'19, fune 30-fuly 5, 2019, Amsterdam, Netherlands

(C) 2019 Copyright held by the owner/author(s)

ACM ISBN 978-1-4503-6789-9/19/06.

https://doi.org/10.1145/3327964.3328490

\section{CCS CONCEPTS}

- Mathematics of computing $\rightarrow$ Spectra of graphs; $\bullet$ Networks $\rightarrow$ Topology analysis and generation; • Computing methodologies $\rightarrow$ Learning latent representations.

\section{KEYWORDS}

Graph distance, topological data analysis, length spectrum, nonbacktracking matrix.

\section{ACM Reference Format:}

Tina Eliassi-Rad. 2019. Length Spectrum Theory, Non-backtracking Cycles, and Two Graph Analysis Tasks. In 2nd foint International Workshop on Graph Data Management Experiences \& Systems (GRADES) and Network Data Analytics (NDA) (GRADES-NDA'19), fune 30-fuly 5, 2019, Amsterdam, Netherlands. ACM, New York, NY, USA, 1 page. https://doi.org/10.1145/ 3327964.3328490

\section{REFERENCES}

[1] S. Soundarajan, T. Eliassi-Rad, and B. Gallagher. A guide to selecting a network similarity method. In SDM, pages 10371045, 2014.

[2] D. Constantine and J.-F. Lafont. Marked length rigidity for onedimensional spaces. Journal of Topology and Analysis, 2018. 